\title{
La perspectiva de género y la relación médico-paciente para el problema de la infertilidad
}

\author{
Gender perspective and the physician-patient relationship in \\ treating infertility
}

\author{
Zoe Díaz Bernal, ${ }^{\mathrm{I}}$ Dailys García Jordá ${ }^{\mathrm{II}}$ \\ ILicenciada en Biología. Máster en Antropología. Profesor Auxiliar. Escuela Nacional \\ de Salud Pública. La Habana, Cuba. \\ ${ }^{\mathrm{II}}$ Licenciada en Biología. Máster en Antropología. Asistente. Facultad de Biología, \\ Museo Antropológico Montané. Universidad de La Habana. La Habana, Cuba.
}

\section{RESUMEN}

Se hace una reflexión acerca de la necesidad de incluir la categoría género en la evaluación de la calidad de la atención a la infertilidad. El punto de partida que se plantea es la identificación de modelos explicativos personales para la infertilidad de hombres y mujeres, y prestadores(as) de salud, para lograr establecer diferencias y similitudes entre sus valoraciones. Se propone volver a pensar en la calidad de la atención desde una perspectiva de género, a partir de la identificación de zonas de encuentros y desencuentros entre los "mundos valorativos" de pacientes y prestadores de salud.

Palabras clave: Género e infertilidad, modelos explicativos de la infertilidad para hombres y mujeres, relación médico-paciente, antropología de la salud.

\begin{abstract}
This paper made a reflection on the need of including the category gender in the evaluation of the quality of care to infertility. The starting point is the identification of personal explanatory models for infertility in men and women, and health providers, with a view to seeing the differences and similarities among their assessments. It made the proposal of re-thinking the quality of care of infertility from a gender perspective, based on the identification of areas of agreement and disagreement between the "evaluating worlds" of patients and of health providers.
\end{abstract}


Key words: Gender and infertility, explanatory infertility models for men and women, physician-patient relationship, health anthropology.

\section{INTRODUCCIÓN}

Para la mayoría de las personas el tener descendencia constituye un elemento de suma importancia, por los múltiples significados sociales y culturales que ello lleva implícito. Sin embargo, no siempre se logra el embarazo o la propia descendencia de manera simple y predecible, lo que obliga a las personas a reformular las expectativas en relación con la paternidad y la maternidad y todo lo que esto implica. Tal es el caso de lo que ocurre ante la infertilidad. ${ }^{1}$

La infertilidad es un problema que enfrenta la humanidad desde tiempos inmemoriales y que tiene importantes connotaciones sobre todo en el orden psíquico y social de los seres humanos. ${ }^{2}$ Suele pensarse que aquellos que la sospechan y a los que muchas veces se les llega a diagnosticar, son los únicos sobre los que hacen diana dichas influencias, sin embargo las personas que comparten espacios sociales con los primeros también la padecen. J

ustamente el concepto de padecer ha emergido con mayor fuerza y claridad de la mano de las corrientes más contemporáneas de la antropología aplicada a la salud. El padecer es la manera en que cada persona sufre las alteraciones de su salud, de acuerdo con su individualidad biológica, psicológica y sociocultural. ${ }^{3}$ Los sustentos del padecer son tres: el cuerpo o biología, la biografía personal y el ambiente donde se desarrollan los seres humanos. De esta manera, los padecimientos o padeceres constituyen uno de los principales fenómenos de construcción de significados, tanto de manera individual como colectiva, ya que los actores necesitan entender, explicar y manejar los procesos que los amenazan e interfieren en sus vidas, y que por demás suelen involucrar a otras áreas de la realidad social. Un ejemplo lo constituye la infertilidad, la cual es vivenciada por las personas que la padecen como un "proceso de profundos dolores y sentires" (sic). ${ }^{4}$

El padecer se expresa de acuerdo a las características de las personas más las del contexto sociocultural en que éstas se desenvuelven. Como consecuencia de la estandarización de la medicina, que a través del modelo biomédico ha "globalizado" las enfermedades, se desconoce la existencia de problemas de salud y padeceres. Las enfermedades se colectivizan por extensión, como lo suelen hacer los epidemiólogos para determinar las prevalencias o incidencias de algunas enfermedades en poblaciones específicas. De acuerdo a los modelos conceptuales de la salud pública es el modelo socio-médico el que exige de la antropología una particular atención en el proceso salud-enfermedad en ambientes sociales, acotados a la familia, comunidad, redes de apoyo social, entre otras organizaciones sociales incluyendo a las sanitarias. ${ }^{5}$

La parentalidad (maternidad y paternidad), enfatizada desde la perspectiva antropológica, debe ser entendida no como un hecho natural, sino como una construcción sociocultural que se encuentra al centro del debate entorno a las consecuencias de la fuerte dicotomía que existe en muchas sociedades, entre la 
masculinidad (asociada al rol de proveedor económico) y la feminidad (destinada al cuidado diario de los hijos). ${ }^{6}$

La procreación y el tener descendencia tienen significados diferentes para cada uno de los miembros de la pareja. Para las mujeres suele ser sinónimo de desarrollo de su papel materno, de protección, afecto y educación, mientras para los varones tiende a tener un significado más dirigido a sentimientos de poder, de patriarcado, de protección, de proveedor, de arribo a la plenitud de la hombría, como ente eminentemente masculino. ${ }^{7}$ Más allá de la reproducción biológica son la reproducción social y los espacios donde esta se desarrolla, quienes determinan en última instancia las percepciones individuales y colectivas de la función reproductiva y la infertilidad.

La infertilidad es algo que rara vez se espera, de ahí que tanto para las personas que la padecen, desde la propia sospecha que los hace acudir a buscar ayuda médica, hasta para los propios prestadores(as) de salud, sea de difícil afrontamiento y manejo.

\section{EL GÉNERO COMO DETERMINANTE}

La necesidad de asumir un enfoque de género en el abordaje de los problemas de salud es tratar precisamente de visibilizar las múltiples formas en las cuales las construcciones de género producen situaciones de inequidad que afectan la salud, y de analizar las normas culturales que "naturalizan" dichas diferencias, haciéndolas parecer inmodificables. En el campo de la salud el desafío radica en demostrar y estudiar cómo el género influye en el proceso salud-enfermedad-atención. Las formas en que mujeres y hombres buscan los servicios de atención de la salud difieren debido a las diferencias de las funciones, oportunidades y expectativas propias de cada sexo. Entre los factores culturales que explican dichas actitudes para las mujeres, se encuentra su participación en las decisiones que afectan a su salud, lo que debe promoverse y potenciarse desde la atención primaria (Las perspectivas de género y la salud pública mundial: consecución de los objetivos de desarrollo acordados internacionalmente, incluidos los Objetivos de Desarrollo del Milenio. Comisión de la Condición Jurídica y Social de la Mujer).

Para el caso concreto de la infertilidad, lo anterior se traduce sobre todo en cierta concepción perpetuada y culturalmente arraigada, en cuanto al responsable papel femenino en la reproducción biológica y social de la especie, en tanto, el binomio mujer = madre persiste en el imaginario social y se refleja en la praxis cotidiana, tanto a nivel interno del grupo como entre grupos heterogéneos. Lo anterior tiene connotaciones en el proceso salud-enfermedad-atención, que no por ciertos, son adecuadamente manejadas por los servicios de salud y que tratarán de destacarse a los largo de este trabajo.

El género interactúa con lo biológico, y un ejemplo de ello es cuando se aplica el enfoque de género a la salud reproductiva, ${ }^{8,9}$ entendido este por el análisis de las relaciones hombre-mujer como dimensión específica de la desigualdad social. Sin embargo, al aplicar la perspectiva antropológica hacia la salud, el término condicionamiento de género define mejor la articulación entre lo biológico y lo sociocultural, al entenderlo como un condicionamiento biocultural_* determinado por el conjunto de atributos culturales asignados según el sexo biológico, que conforman una manera particular de vida y de concepción del mundo, o lo que es lo mismo, una subjetividad diferente para hombres y mujeres, la construcción de una identidad excluyente de lo masculino y lo femenino, a partir de la apropiación 
consciente o inconsciente de un universo de valores, prácticas, lenguajes, símbolos y formas de aprehender al mundo, lo que condiciona la reproducción cultural. ${ }^{9}$ De tal manera, a nivel colectivo suele prevalecer el criterio de que la infertilidad obedece a causas de origen femenino, como si la concepción de un embarazo fuera responsabilidad exclusiva de las condiciones biológicas femeninas.

Cada día más, las investigaciones en el campo de las ciencias de la salud, reclaman por una perspectiva de género que articule a su vez, una posición y una intencionalidad de un sujeto (mujer u hombre), lo que constituye tanto "un punto de vista" como una selección y delimitación del campo de lo observado. La investigación con perspectiva de género se interroga a si misma respecto a los temas importantes de ese sujeto, es decir, respecto de la significación que para mujeres y hombres tiene la diversidad de situaciones, relaciones, experiencias y vivencias de una realidad específica.

Diferentes estudios han demostrado que son múltiples los factores que inciden y determinan que una persona acuda a los servicios de salud ante un problema de salud, ${ }^{10}$ entre ellos se encuentra la edad, el sexo y la propia naturaleza de los síntomas, lo que a través del análisis del marco antropológico, ${ }^{11}$ responde a los capitales de la persona, que embebidos en sus tres niveles: macroentorno, mesoentorno y microentorno, sustentan los modelos explicativos de la enfermedad. El macroentorno se refiere el ámbito más general en que se inserta el ser humano (cultura, ideología, religión, sistema de derechos y base económica). El mesoentorno comprende al ámbito más cercano y concreto de la población analizada (ubicación geográfica, procesos económicos de producción-distribuciónconsumo, instituciones y organizaciones sociales). Por último, el microentorno está representado por el espacio inmediato al individuo (escenario donde se construye la cultura cotidiana).

En el interior de estas tres dimensiones se encuentra la persona, su biología (cuerpo), genética, sexo, su biografía personal y sus capitales económico, social y simbólico, eje a partir del cual partirán todos los análisis posibles del problema. Partiendo de ello no podrá analizarse el modo en que se concibe y vive la infertilidad de manera homogénea y universal, como tampoco podrá esperarse que las alternativas de atención buscadas y la propia atención brindada, obedezcan a patrones preestablecidos. Aplicado al género, aún cuando se comparta por la pareja infértil, espacios comunes a nivel del macro y mesoentorno, las relaciones de pareja y las funciones de género, asociados al cuidado y mantenimiento del hogar y los hijos, asignados comúnmente a las mujeres, marcarán diferencias sentidas y vividas desde lo individual y a nivel social. Por otra parte, los capitales personales, específicamente el capital social y el simbólico, tendrán una expresión diferencial para mujeres y hombres, en dependencia del factor de infertilidad (femenino/masculino/ambos) y sus respectivas parejas.

Aunque existe un reconocimiento creciente acerca de la criticidad en la determinación de la salud, tanto del componente social como del biológico, el debate entorno a la sobre morbilidad femenina y la sobre mortalidad masculina, refleja que persisten tensiones entre quienes priorizan a uno sobre otro y viceversa. La idea del mayor "malestar de las mujeres" ha tenido explicaciones desde lo biológico y más recientemente desde su determinación social. Una explicación podría ser que las mujeres son más dadas a manifestar o expresar su malestar que los hombres o, que las condiciones materiales de vida y sus funciones (materno, doméstico, sexual y otros) conllevan a una mayor morbilidad asociada. Los problemas que presentan los hombres en su perfil epidemiológico, igualmente han sido vinculados a las relaciones de género, sobre todo a aquellos que representan 
los estereotipos de masculinidad: represión de emociones, temor a ser visto como débil y agresividad. ${ }^{8}$

La OPS a finales del siglo pasado, reconoció que en todos los países de la región es mayor el número de hombres que de mujeres que mueren por cáncer, enfermedades cardíacas, episodios cerebrovasculares y accidentes. En cuanto al valor que esto tiene para la atención médica y su propia búsqueda, se ha afirmado que el sesgo que ello produce para la atención, refuerza y no alivia el problema subyacente y que los hombres pueden estar inhibidos para percibir síntomas, buscar atención y cumplir con las indicaciones médicas. ${ }^{8}$

La inequidad en las relaciones de pareja es expresión de las desigualdades de poder determinadas por el condicionamiento de género, formuladas fenoménicamente en las funciones y estereotipos que le caracterizan, lo que se refleja en los comportamientos ante el propio embarazo o ante la imposibilidad de lograrlo. La cultura patriarcal excluye al hombre de sentimientos y comprometimientos afectivos, lo que influye en que los varones mantengan una distancia durante el proceso de diagnóstico y tratamiento de la infertilidad, "protectora" de la repercusión social que ello representa para su masculinidad y de los sentimientos de inferioridad que les genera, ${ }^{12}$ mientras que la mujer se involucra con repercusión para su autoestima, satisfacción personal y salud mental. ${ }^{13}$

Parte del matiz que aporta el género a la atención de la infertilidad, radica en la responsabilidad asignada y asumida en cuanto al seguimiento de dicha atención para y por las mujeres. Aún cuando en los propios protocolos de atención se refiere la necesidad de concurrencia al servicio en pareja, y no de uno u otro miembro por separado, es la mujer quien recibe las órdenes e instrucciones médicas y quien posee historia clínica para el control por parte de la institución de salud. Por otro lado, sobre la mujer gravitan presiones sociales y familiares que no inciden de igual manera sobre los hombres, para quienes hay más tiempo para ser padres, el cese de la capacidad reproductiva femenina impone sin dudas un derrotero para ellas.

Téngase en consideración que la sinonimia establecida entre mujer infértil y mujer vacía, parte de la condición de madre que se espera de toda mujer, papel asignado y asumido para y por la hembra humana a quien "le corresponde" reproducir la especie, lo que socialmente y desde la pareja es además garante de estabilidad familiar y vital para la no disolución de la vida en pareja. Al interior de la pareja esto puede conducir a crisis tanto en el orden emocional como psíquico y sexual, lo que puede resultar una relación de pareja disfuncional, cargada de culpas y en muchos casos contaminada con relaciones extramatrimoniales, que sobre todo para el hombre que busca la reafirmación de su condición viril. Todo ello refuerza que, desde la propia pareja, sea la mujer quien sienta mayor responsabilidad en la búsqueda de atención y su seguimiento, así como que la infertilidad se visualice como un evento femenino, aún cuando sea masculina.

\section{La infertilidad para las mujeres}

Históricamente a la mujer se le han asignado, incluso de forma mítica, binomios inseparables tales como que mujer es igual a madre, o que mujer es igual a familia, lo cual ha definido algunos mitos femeninos, y que influye en todas las esferas vitales femeninas y organiza su vida independientemente de cualquier condición. ${ }^{14}$ A la mujer se le educa para la casa, asumiendo papeles de buena madre y esposa, los que conforman el eje de la subjetividad femenina. Con la llegada de un embarazo la mujer deja de serlo para convertirse en madre, muchas veces en detrimento del propio papel de mujer, todo lo que trae aparejado de por sí

http://scielo.sld.cu 
implicaciones negativas en la sexualidad femenina, pues se enfatiza y refuerza el tradicional binomio mujer $=$ madre. ${ }^{15}$

Lo femenino socializado en el modelo de la pasividad, la ternura, el afecto, la complacencia, la maternidad y la valoración del ser mujer a partir de las tareas del maternaje ${ }^{16}$ supone un estilo de vida de un número de mujeres. Cuando la función mujer = madre no se puede lograr, daña su autoestima y provoca depresión. Es entonces cuando ante la imposibilidad del embarazo por infertilidad se sienten frustradas, pues dejan de cumplir con una de sus principales funciones y no satisfacen desde lo sociocultural y lo personal uno de los principales arquetipos femeninos.

El maternaje se conceptualiza como todas las acciones o tareas asociadas con el proceso de maternidad y se manifiesta en comportamientos específicos: pérdida de horas de sueño, posposición del disfrute y de sus propias prioridades en aras del cumplimiento de la función maternal, entre otros. El maternaje como comportamiento asignado y asumido cultural y socialmente para las mujeres, es un proceso interminable, que remite a una larga cadena de sucesos asociados con la maternidad, que solo termina con la vida de la mujer y en el cual ella juega un papel protagónico, ${ }^{17}$ el maternaje como constructo sufre modificaciones ante la imposibilidad de procrear, de hecho muchas mujeres infecundas se sienten vacías y frustradas en su condición femenina. ${ }^{13}$

\section{La infertilidad para los hombres}

Específicamente para los varones la paternidad es una parte fundamental de la identidad masculina, la cual opera como un elemento estructurado de deber en su ciclo de vida, pues a este nivel el varón se enfrenta a desafíos/mandatos, entre los que se destacan: trabajar, formar una familia y tener hijos. ${ }^{718}$

Estudios realizados en algunos países de América del Sur plantean que, aunque existen diferencias en las prácticas, la paternidad es un eje central de la masculinidad, la cual se vive como el momento en que se cierra el período juvenil, lo que significa un reordenamiento en la vida del varón y su inserción en un nuevo período en el que obtiene pleno reconocimiento social. Es el punto en el que el varón se convierte en adulto al adquirir una identidad pública como representante de su grupo familiar, pues completa así los requisitos para ser considerado un adulto completo..$^{6,7}$

Sin embargo, aunque la experiencia de ser padre se define como una expresión de deseo profundo y como parte del proyecto de vida para la mayoría de los hombres, esta no se deja a la libre elección, sino que existe una intensa presión social dirigida a forzar a los varones a tener hijos, ya que se tendería a dudar de la hombría de aquellos que no cumpliesen ese mandato. ${ }^{1}$

La paternidad no es percibida por los varones y la sociedad en general, solamente como una confirmación de la última prueba de virilidad masculina, al garantizar que pueden fecundar a una mujer, sino que su interpretación también les garantiza a ellos la existencia de un espacio de patriarcado dentro del contexto familiar, pues se convierten en jefes de la familia, en la autoridad del hogar, y su trabajo permite cubrir las necesidades de la familia y de los hijos. ${ }^{7}$ Por eso la infertilidad podría entrañar en los varones sentimientos de inferioridad, ${ }^{12,19}$ dado que su valor como varón se vería limitado por no poder construir la familia anhelada, y de este modo crear un sistema de jerarquía donde él jugaría el papel principal. Si dichas funciones se vieran anuladas por cualquier razón, y principalmente si es por 
razones ajenas a su voluntad, bien por causas de origen anatomofisiológico de él o su pareja, puede ser bastante alta la probabilidad de que se vea afectada su autoestima, incluso con capacidad de afectación para la estabilidad de pareja y la familia, llegando hasta el punto de la disolución de la relación primera. Todo ello estaría aparejado por supuesto, a un potencial cuestionamiento desde lo social, a nivel colectivo dentro del grupo (entre los hombres) y entre los grupos (en relación con las mujeres), de sus capacidades como varón.

Sin embargo y aún cuando no se ha explicitado así desde las investigaciones más recientes entorno al tema, aunque como ya se ha dicho son escasos los estudios de este tipo dirigidos a la población masculina, debe explorarse la construcción de la paternidad para los hombres, también desde el deseo de ser padres en virtud de trascender, de participar en la formación de algo tan sublime como lo es un nuevo ser humano, aunque posiblemente la vivencia de dicho constructo se produzca a una edad diferente, más desplazada en el tiempo, con respecto a las mujeres. De esa manera, estamos planteando que al margen de la necesidad de un espacio de patriarcado a lo interno de la familia y de una necesidad de reconocimiento como entes viriles y heterosexuales a nivel social, lo puramente emocional y afectivo puede expresarse también en el deseo de los hombres por ser padres.

Según otros autores, dentro del sentido "natural" que le atribuyen los varones cubanos a la paternidad, la dimensión biológica ocupa una jerarquía superior sobre la dimensión social, de esta manera un hijo no es considerado como tal si no existe vínculo biológico, lo cual ha sido comprobado a través de los guiones discursivos de algunos hombres entrevistados. ${ }^{1}$ Lo anterior mueve el pensamiento hacia la relación de causalidad e influencia del entramado sociocultural del que se originan y donde se desarrollan esos hombres, de otras variables socioeconómicas y de la cultura cubana de la que son hijos.

Como parte del sentido que le atribuyen los varones a la procreación, parece ocupar un importante lugar el embarazo o preñez de la mujer, el que es percibido como un símbolo para la sociedad (incluida su compañera) que argumenta y denota de manera incuestionable su virilidad y su capacidad reproductiva, además de demostrar socialmente su orientación heterosexual activa, al haber sido capaz de penetrar a su compañera y, como resultado, embarazarla. La necesidad constante de demostrar la heterosexualidad masculina es un elemento que reviste una gran importancia teniendo en cuenta el modelo de masculinidad hegemónica en la etapa adulta del varón. ${ }^{1}$

Individuos entrevistados por Calero y Santana, ${ }^{4}$ se sienten padres, no meramente por el hecho de criar y mantener a un niño, sino porque este infante tiene vínculos genéticos con su progenitor que demuestra el parecido. Por tanto, si el hijo no es concebido bajo procedimientos considerados como normales, este símbolo pierde sentido y autenticidad ante la sociedad, en la cual su compañera se encuentra incluida. De ahí el rechazo mayoritario de algunas de las técnicas de reproducción asistidas por parte de los varones, sobre todo cuando no implica la relación coital o se trata de la inseminación heteróloga.

La paternidad constituye uno, tal vez el más importante, de las funciones sociales y culturalmente esperados por los varones en la adultez, por lo que la infertilidad funcionaría como un fenómeno que obstaculiza el logro de esta condición, la cual se traduce en padecimiento. Así, la negatividad que gira entorno a los significados que le atribuyen los varones a la infertilidad se enmarca, se construye y se vivencia como "potencialidad reproductiva anulada" (sic) ${ }^{1}$ no sólo en términos de la capacidad de embarazar a una mujer, sino en cuanto a la incapacidad de satisfacer una demanda construida desde el género. ${ }^{1,20}$ 
De acuerdo con esto, el entendimiento, explicación y manejo que realizan los varones junto a sus compañeras sobre su padecimiento los conduce a la búsqueda de alternativas para darle solución al problema que enfrentan y vivencian, 0 al menos que dichas alternativas minimicen las consecuencias negativas que este padecimiento les produce. Ha sido informado que la selección de una u otra alternativa de solución estará regulada y modulada por el valor de pérdida asociado a la posibilidad de logar un embarazo que dicha alternativa representa para la naturalidad de la concepción, referido al hecho de concebir el embarazo como producto de una relación coital, ${ }^{4}$ lo que se encuentra a su vez; estrechamente relacionado a las características masculinas del varón dentro del modelo hegemónico de masculinidad. ${ }^{21}$

Por su parte, la adopción de un niño es la alternativa al problema de la infertilidad que cuenta con menos popularidad por parte de los varones. ${ }^{1,19}$ La selección de la adopción funciona más bien como una variante a la resignación.

Según algunos autores, la práctica de creencias religiosas o sincréticas en Cuba por parte de los varones es baja, quienes en su mayoría utilizan la santería como culto sincrético, lo que desde el punto de vista cultural se corresponde con las tradiciones legadas por sus ancestrales inmigrantes africanos. ${ }^{1}$

En estudios recientes se encontró que resulta difícil encontrar varones que se muestren resignados a vivir una vida futura sin hijos, por el contrario, ellos mantienen con mucha fuerza la idea de poder lograr, por la alternativa que fuera (incluida la adopción), la posibilidad de tener un hijo. Lamentablemente una de las alternativas referidas por los varones y utilizadas ante la desesperación y desasosiego que genera la imposibilidad de tener hijos, se dirige a la disolución definitiva del vínculo matrimonial o consensual, en la esperanza de lograr el hijo anhelado con otra compañera. ${ }^{4}$

El varón infértil suele ser presa de angustias y depresión porque equipara su virilidad con el hecho de ser padre. Muchos hombres se muestran reacios a enfrentar el problema o temen que se conozca públicamente, se niegan a la realización de cualquier estudio (ej. espermograma), a asistir a consulta pues lo consideran "cosas de mujeres", y lógicamente esto hace que surjan tensiones, conflictos en el matrimonio o en la relación convivencial. ${ }^{7}$

Entre las disfunciones sexuales que pueden aparecer en los varones está la eyaculación retardada en la cual hay una falla de eyaculación intravaginal, lo cual imposibilita el embarazo. Esto puede aparecer como causa de la infertilidad o puede ser como reacción o respuesta complicada al tratamiento, o la reacción de la pareja estéril. También podría aparecer o ya existir una eyaculación retrógrada, durante la cual el líquido seminal es enviado hacia la vejiga (esta enfermedad puede ser el resultado de diabetes mellitus, trastornos neurológicos, uso de ciertos fármacos, intervenciones quirúrgicas que afecten la inervación vesical, entre otros). La disfunción sexual eréctil puede causar esterilidad cuando resulte imposible el coito, pues o no puede lograrse nunca o se logra rara vez en la fase no fértil del ciclo menstrual, pero esta disfunción no constituye causa frecuente de infertilidad, cuando aparece, mayormente resulta como respuesta al tratamiento de la infertilidad, o porque hay que tomar muestras del semen, lo que inquieta y preocupa al hombre, y como respuesta surgen imposibilidades de la erección. ${ }^{14}$

La disfunción sexual eréctil se puede encontrar formando parte del "matrimonio no consumado", donde hay un vaginismo como sustrato, por lo ha existido imposibilidad para realizar el coito. Esta disfunción forma parte de este complejo cuadro, que con adecuada ayuda puede solucionarse. 
La feminidad tradicional puede provocar conflictos en mujeres con problemas de fertilidad y habría que explorar si existen variaciones según se deba a factores masculinos o femeninos. Por otro lado, las manifestaciones de poder intragenéricas puede que se aprecien mejor a través de la violencia simbólica que puede representar la presencia o ausencia de hijos, entre varones o entre mujeres. Estos son fenómenos que se relacionan desde la reproductividad, para afianzar o no la identidad de género en las mujeres. Los varones tienen a su vez la posibilidad de replantear su masculinidad o no desde la reproductividad. Aunque existen diferentes teorías respecto de la construcción de las masculinidades no puede negarse que, al menos, la pareja que afronta un problema de fertilidad se plantea de nuevo la conceptualización de la masculinidad y la feminidad propia, de la pareja y de los otros. Si se transforma en un problema o no, depende del vivenciar en pareja. Sin embargo, una constante cultural es la necesidad de demostrar la virilidad que puede traducirse en la expresión de la paternidad, se es plenamente masculino en el momento en que se reproduce. Esto explicaría de alguna manera el por qué aparece más ansiedad en los varones que en las mujeres ante los problemas de fertilidad y es de alguna manera la expresión del machismo. ${ }^{23}$

\section{LA RELACIÓN MÉDICO-PACIENTE}

Los individuos implicados en la infertilidad suelen argumentar que la atención que les ofrecen los proveedores de salud se posiciona en una especie de "frialdad médica", que se dirige básicamente a la atención de la infertilidad como enfermedad y no a la atención de la pareja infértil, como personas que vivencian un padecimiento, de hecho es la demanda de hombres y mujeres con problemas de infertilidad y esterilidad la imposibilidad de tramitación en la consulta médica para el dolor que los envuelve; de manera que la atención se centra casi exclusivamente en los aspectos biológicos del problema presente y no establece un balance de estos aspectos con los de tipos psicológicos y sociales que dicho padecer les genera. ${ }^{4,24}$ También han referido que los médicos los mantienen al margen de lo que ocurre, al no brindarles de manera clara las explicaciones pertinentes, que les permitan conocer y comprender el por qué de los estudios a los que deben someterse y las alternativas o tratamiento a seguir para solucionar dicho padecimiento; señalan además, que las indicaciones brindadas por los médicos se dirigen hacia lo que deben hacer o al medicamento que deben tomar, sin dejar un espacio abierto para una reflexión y educación conjunta entre proveedores y pacientes.

A lo anterior se adiciona el hecho de que la mayoría de los afectados coinciden al considerar que las consultas de estudio y seguimiento que brindan los proveedores de salud son muy distantes y espaciadas en el tiempo, lo que hace más prolongada la espera para la obtención de resultados satisfactorios, y algunos justifican la percepción de demora detrás de los niveles de ansiedad que la espera les produce. Otros no la justifican, sino que perciben esa demora como un elemento que atenta y refuerza el dolor que siente la pareja. ${ }^{4}$

Una particularidad de la búsqueda de atención a la infertilidad en el contexto cubano, es el abandono en más de una ocasión del servicio al que se está acudiendo, lo que consecutivamente de manera inmediata o mediata conlleva al cambio hacia otro. Lo anterior parece deberse a la necesidad de hallazgo de una atención más empática, cálida y por tanto efectiva, según los criterios valorativos de los usuarios, quienes al ver pasar el tiempo sin respuestas, llegan a pensar que su problema no tiene solución y esa es una respuesta que no desean escuchar. De tal manera algunas mujeres han pasado por la experiencia de la misma prueba 
diagnóstica en más de una ocasión, sin saber de antemano que así es, pues nunca supieron bien el nombre de la misma y el por qué de su indicación. Dichas pruebas además de tener idénticos resultados han sido invasivas y dolorosas.

Para lograr una atención adecuada a la infertilidad y contribuir a su solución o manejo, es necesario ante todo un enfoque empático hacia los individuos y las parejas con dicho problema. Tradicionalmente la atención brindada por los prestadores de salud ha tenido un carácter eminentemente biologicista, ${ }^{25}$ ya que para la biomedicina el no ser capaz de reproducirse suele ser una anormalidad o limitación que necesita ser corregida. ${ }^{26}$ Como para cualquier otra dolencia o enfermedad, el sistema médico ha establecido algoritmos de trabajo para la atención, estudio y tratamiento de la infertilidad: el tratamiento clínico-

farmacológico antecede al quirúrgico, que se utiliza como tratamiento alternativo o complementario, dirigido básicamente a corregir efectos o defectos anatómicos que interfieren con la fisiología del aparato reproductor (masculino o femenino) ${ }^{1,20}$

El modelo médico hegemónico continúa concibiendo a la infertilidad como un trastorno puramente físico, cuya solución está a la espera de la terapéutica médica o quirúrgica adecuada, aún cuando se ha tenido que reconocer que las parejas infértiles experimentan alteraciones emocionales importantes y su impacto puede llegar a ser peor sobre la función y satisfacción sexual, todo lo cual pretende ser justificado por lo prolongado de los estudios y tratamientos.

A diferencia de muchos países del mundo, el sistema de salud cubano alcanza a todos los miembros de la población por su gratuidad, accesibilidad geográfica y alta calificación, lo cual hace que las personas acudan primeramente a estos servicios con el fin de solucionar sus malestares y padeceres. Sin embargo, hay que reconocer que el carácter biologicista y medicalizador de la atención en salud, la relación asimétrica en el vínculo médico-paciente, la participación subordinada y pasiva de los pacientes en las acciones de salud, la producción de acciones que tienden a excluir al paciente del saber médico y la tendencia al control social e ideológico, características todas del modelo médico hegemónico, ${ }^{27}$ hacen que las personas implicadas en el padecimiento concreto de infertilidad, establezcan un análisis de las potencialidades y deficiencias que ellos le atribuyen al sistema y a las alternativas de solución que dicho sistema sugiere. Una de las principales consideraciones que expresan las parejas infértiles en relación con la atención brindada por los profesionales de la salud, se refiere precisamente a la medicalización de la atención y a la posición de "frialdad médica" de los médicos que se dirigen básicamente a la atención de la infertilidad como enfermedad y no a la atención de la pareja infértil como personas que padecen.

La categoría género ha sido utilizada en la evaluación de la calidad de la atención fundamentalmente en dos áreas: la de salud sexual y reproductiva y la de violencia contra la mujer. La evaluación contemporánea de la calidad de los servicios ha asumido lo que algunos han dado en llamar "nuevo paradigma" y que introduce la llamada "satisfacción de los clientes internos" (prestadores de salud) y "satisfacción de los clientes externos" (los usuarios de los servicios). ${ }^{8}$ Hasta el momento, las posibilidades de analizar las opiniones del cliente externo aplicando una perspectiva de género, han sido insuficientes, ya que se ha restringido al nivel de opinión, sin lograr analizar cómo se construyen socialmente dichas opiniones, lo que requiere de la aplicación de técnicas propias de la investigación cualitativa.

Desde una perspectiva antropológica, la idea de los modelos explicativos aportada por Kleinman, ${ }^{28}$ permite evaluar el grado de eficacia de la atención médica y los problemas en general de la calidad de la atención, así como identificar el conjunto de ideas vigentes para grupos particulares en contextos sociales específicos, en 
relación con problemas de salud, padeceres y enfermedades concretas. La aplicación de los modelos explicativos en las evaluaciones de este tipo tiene dos ventajas sustanciales, una es que permite abordar simultáneamente dos perspectivas: la de usuarios/as y prestadores/as y la otra es que permite incorporar las ideas de los actores sociales acerca del proceso de salud-enfermedad, posibilitando el conocimiento acerca del modo en que se construyen dichas valoraciones ${ }^{11}$

Las formas en que cada persona explica los problemas de salud y/o las enfermedades, ya sean propios o ajenos, está influenciado por un complejo entramado socio- cultural, lo que determina sus percepciones y valoraciones acerca del proceso salud-enfermedad y de la atención a este. Lo anterior obliga al despojo de todo juicio de valor, desde el punto de vista biomédico, de las explicaciones individuales aportadas, las que estarán indisolublemente determinadas por sus biografía individuales y las de su entorno social, ${ }^{11}$ lo que Vargas y Casillas (2004) denominaran biografía personal y ambiente, y que conjuntamente con el cuerpo o biología constituyen los sustentos del padecer.

Cabe hacer la salvedad en este punto de la diferencia existente entre términos que erróneamente suelen emplearse indistintamente: padecer y enfermedad, el primero es la manera en que cada persona sufre las alteraciones de su salud, de acuerdo con su individualidad biológica, psicológica y sociocultural, mientras que la enfermedad está asociada a una entidad nosológica concreta, identificable por los métodos/técnicas diagnósticos. ${ }^{3}$ Así, existirá un grupo de personas infértiles para los que estar privados de la posibilidad de concebir, sea similar a dejar de existir como personas felices, capaces de llevar una vida armónica. Para otras sin embargo, este será un escollo que una vez superado no lastrará las realizaciones humanas y expectativas futuras. He aquí un ejemplo de cómo un mismo problema de salud puede padecerse de dos maneras diferentes, aún cuando obedezcan a idénticas causas. Por supuesto, el género marcará una diferencia en tal sentido, pero como ya se ha dicho, el padecer tiene otros sustentos no menos importantes.

El punto de partida para el caso que nos ocupa debe ser precisamente la identificación de los modelos explicativos para la infertilidad de hombres y mujeres, y sus consecuentes valoraciones de la atención, lo que expresará diferencias y similitudes en las valoraciones que estos hacen, así como diferencias y similitudes de ambos grupos con respecto a los prestadores de salud. En tal sentido opinamos que el abordaje de "mundos valorativos" propuesto por Pittman. ${ }^{8}$ se ajusta mucho mejor a los estudios de calidad de la atención desde una perspectiva de género que la categoría "relación- interpersonal" frecuentemente empleada, ya que como bien plantea dicha autora, esta última da a entender que los problemas tienen un carácter individual y no social. Por otra parte, a la hora de evaluar la relación médico-paciente atravesada por el género, suele mirarse hacia los puntos de desencuentro entre los mundos valorativos de dichos actores y al igual de lo que opina Pittman, opinamos que resultaría mucho más esclarecedor descubrir los puntos coincidentes que remarcarán las brechas que puedan establecerse en la atención, dadas las perspectivas de género de los implicados: médico(a)-pacientes.

Habría que preguntase entonces, si la atención a la infertilidad se presta, aún estando establecidos los protocolos de atención, de igual manera por mujeres que por hombres prestadores del servicio, y si la relación médico-paciente que se establece es similar, si adicionado a lo anterior, el factor de infertilidad fuera masculino o femenino. Precisamente hablando de los puntos de encuentros entre los mundos valorativos de pacientes y prestadores(as) de salud, podría estarse reforzando las asignaciones de género que en definitiva, gravitarán sobre el padecer de la infertilidad, de una manera u otra.

http://scielo.sld.cu 
Con todo lo que hasta aquí se ha expuesto, emerge por si misma la principal conclusión, y es que las investigaciones, políticas, sistemas y servicios en el campo de la salud, requieren de una perspectiva de género, que aportaría en gran cuantía a la visión médico-social de los problemas de salud a la que está siendo llamada la salud pública cubana. La atención a los puntos de encuentro y desencuentro entre los mundos valorativos de prestadores de salud y pacientes, permitirá elevar la calidad y calidez de la atención a la infertilidad, evitando que se ensanchen las brechas existentes en el proceso salud-enfermedad-atención, dadas las perspectivas de género de prestadores de salud y usuarios de los servicios.

\section{REFERENCIAS BIBLIOGRÁFICAS}

1. Calero JL, Santana F. La infertilidad como evento de frustración personal. Reflexiones de un grupo de varones de parejas infértiles. Rev Cubana Endocrinol. $2006 ; 17(1)$.

2. Fernández A, Rivero R. Trastornos de la fertilidad en el municipio "Arroyo Naranjo". Rev Cubana Obstet Ginecol. 1997;23(1).

3. Vargas LA, Casillas LE. Perspectiva antropológica de la participación comunitaria para la promoción de salud. En: García Viveros M, editor. Salud comunitaria y promoción de la salud. Las Palmas de Gran Canaria: Instituto Canario de Estudios y Promoción Social y Sanitaria; 1999. p. 131- 45.

4. Calero JL, Santana F. La solución ante la infertilidad. Representaciones de un grupo de varones atendidos por este padecimiento. Rev Cubana Endocrinol. $2006 ; 17(2)$.

5. Reverte Coma JM. La tesis doctoral en Antropología Médica, el trabajo de campo. Revista EL MÉDICO. 1988(270):69-78.

6. Fuller N. Masculinidades. Cambios y permanencias. Lima: Fondo Editorial de la Pontificia Universidad Católica del Perú; 2001.

7. Olavarría J. Ser padre en Santiago. En: Fuller N, editor. Paternidades en América Latina. Lima: Fondo Editorial de la Pontificia Universidad Católica del Perú; 2000. p. 74-100.

8. Pittman P. Género y calidad de atención: el caso de hipertensión y diabetes en Avellaneda, provincia de Buenos Aires, Argentina. Washington, D.C.: OPS; 1999.

9. Artiles L. Contribución de los roles de género en la determinación del síndrome climatérico [tesis]. La Habana: Instituto Superior de Ciencias Médicas de La Habana; 2001.

10. Sáez M. Modelo clínico centrado en el paciente. Arch Med Fam Gen. 2008;5(1).

11. Artiles L. Marco antropológico de los determinantes sociales de salud como herramienta para un enfoque personalizado de las Infecciones de Transmisión Sexual y el VIH-SIDA. Seminario Internacional Problemas de la Población Mundial en el Siglo XXI: Dilemas y Desarrollo. La Habana: Asociacion Cubana de las Naciones Unidas; 2006.

http://scielo.sld.cu 
12. Family Health International. Los hombres contribuyen a la infertilidad y sufren a causa de ella. Network en español. 2004;23(2):17-20.

13. Kolodny RC, Johnson VE. Tratado de medicina sexual. 1985:309 (Edición Revolucionaria).

14. Ares MP. Genero, salud y cotidianidad. La Habana: Editorial Científico Técnica; 2000.

15. González I. La infertilidad, el maternaje frustrado. Rev Cubana Med Gen Integr. $2002 ; 18(3)$.

16. Alfonso CA. Género salud y cotidianidad. La Habana: Editorial Científico Técnica; 2000.

17. González LI. El proceso reproductivo. Algunas consideraciones sobre el maternaje. Rev Cubana Med Gen Integr. 2001;17(5).

18. Olavarría J. Y todos querían ser (buenos) padres. Varones de Santiago de Chile en conflicto. Santiago de Chile: Serie Libros FLACSO-Chile;2001.

19. Calero JL, Magallanes M, Rechkemmer A, García M. Infertilidad o paternidad frustrada. Significados de este fenómeno desde la perspectiva masculina peruana. Rev Cubana Salud Pública. 2003;29 (Suppl 1):48.

20. Andrews F, Abbey A, Halman LJ. Stress from infertility, marriage factors and subjective well-being of wives and husbands. J Health Social Behaviour. $1991 ; 32: 238-53$.

21. Connell RW. La organización de la masculinidad. En: Valdez TY, Olavarría J, editores. Masculinidades: poder y crisis. Santiago de Chile: FLACSO Chile;1997.

22. Álvarez L, Calero JL, León ME. Percepciones y comportamientos de la sexualidad y la reproducción según los hombres. La Habana: Publicaciones Azucareras; 2004.

23. Álvarez-Díaz J. Sexualidad en parejas con problemas de fertilidad. Gac Méd Mex. 2007;143(1).

24. Jadur S. La infertilidad y sus modos de sufrimiento. Un dolor puro de esterilidad. República Argentina. P. 12. Año 2002, diciembre, 1. Columna de Psicología. Disponible en: http://www.pagina12.com.ar

25. Calero JL. Salud reproductiva: ¿Estamos educando bien? Rev Sexol Soc. $1999 ; 5(3): 33-5$.

26. Castañeda $\mathrm{E}$, Bustos $\mathrm{HH}$. La ruta del padecer en mujeres con diagnóstico de infertilidad. Perinatol Reprod Hum. 2001;15(2):124-32.

27. Menéndez E. El punto de vista del actor: homogeneidad, diferencia e historicidad. En: Menéndez E, editor. Relaciones de Estudios de Historia y Sociedad. México: El Colegio de Michoacán; 1997. p.239-69.

28. Kleiman A. Patients and healers in the context of culture. Berkeley: University of California Press; 1980.

http://scielo.sld.cu 
* Este concepto se desarrolla sobre la base del de proceso biocultural que describen Luis Alberto Vargas y Leticia Casillas como aquellas situaciones derivadas de la fisiología humana modificadas y matizadas por la cultura. Vargas $L A$ y Casillas $L$. Perspectiva antropológica de la participación comunitaria para la promoción de la salud, conferencia presentada en el Primer Coloquio Nacional de Antropología y Psicología de la dirección Nacional de Etnología y Antropología Social del Instituto Nacional de Antropología e Historia. México: 27 septiembre al 1 de octubre, 1999. En consulta con Vargas me propuso denominarlo como condicionamiento biocultural de género el que se expresa en la asignación de roles, en la construcción de estereotipos para cada sexo, en la representación del ser mujer y el ser hombre que se conforma en el contexto cultural donde se forma el sujeto.

Recibido: 30 de junio de 2009.

Aprobado: 30 de julio de 2010.

Zoe Díaz Bernal. Escuela Nacional de Salud Pública. Calle Línea esq. I, El Vedado 10400. La Habana, Cuba. Correo electrónico: zoe@ensap.sld.cu;

zoe.diaz@infomed.sld.cu 